\title{
Genetic Instability Is Not a Requirement for Tumor Development
}

\author{
Walter Bodmer \\ Cancer and Immunogenetics Laboratory, Weatherall Institute of Molecular Medicine, University of \\ Oxford
}

\section{Introduction: Arguments for a Requirement for Genomic Instability}

The idea, originating in the early years of the 20th century, that each cancer is an independent somatic evolutionary process has been abundantly confirmed by modern molecular approaches (1-3). Genetic and epigenetic changes that increase the chance of outgrowth of the developing tumor are successively selected for.

It has, however, often been argued that normal mutation rates are not sufficient to explain the number of steps that seem to be needed for giving rise to a cancer. This has led to the hypothesis that there is a requirement for genomic instability in the somatic evolution of sporadic cancers. The instability is assumed to be due to an increase in gene mutation rates or to an increase in the rate of production of chromosomal abnormalities (4-6). In apparent support of this hypothesis is the observation of increased mutation rates in certain types of sporadic cancers due to the presence of mutations in genes involved in DNA repair, and the fact that a high proportion of cancers, especially the common carcinomas, have grossly deranged karyotypes. The argument for genomic instability, however, seems to ignore the power of natural selection, which is so fundamental to any process of Darwinian evolution.

\section{The Power of Natural Selection}

The contrast between the arguments for genetic instability, as opposed to the power of natural selection, is analogous to the arguments between De Vries, a rediscoverer of Mendelian inheritance at the turn of the 19th to 20th century, and the Darwinians. De Vries argued that evolution was driven by mutation not selection. He was led to this idea because he saw an apparently high mutation rate in the plant Oenothera he was working with. This, however, turned out to be due to recombination between chromosomes in a highly convoluted karyotype. RA Fisher, on the other hand, who was my PhD teacher and one of the founders of population genetics based on Mendelian principles, showed that quite small selective advantages and very low mutation rates could readily account for the evolutionary process (7), and I believe that the same is true for cancers. It seems strange that a lack of appreciation of the power of natural selection should again turn up in this modern context in relation to cancer.

I will now review the wide range of arguments against a need for genomic instability for the development of cancers and provide an explanation for why, nevertheless, genetic instability of cancers is often seen.

\footnotetext{
(C)2008 American Association for Cancer Research.
}

Requests for reprints: Walter Bodmer, Cancer and Immunogenetics Laboratory, Cancer Research UK/Weatherall Institute of Molecular Medicine, Oxford OX3 9DS, United Kingdom. Phone: 44-0-1865-222422; Fax: 011-44-0-1865-222431; E-mail: walter.bodmer@hertford.ox.ac.uk.

Disclosure of Potential Conflicts of Interest

No potential conflicts of interest were disclosed. 


\section{Arguments against a Requirement for Genomic Instability Mismatch repair mutations do not generally occur before APC changes}

The most striking readily observed change in mutation rate in human cancers is due to the occurrence of mutations in, or epigenetic silencing of, one of the genes controlling repair of mismatched (MMR) DNA lesions, primarily $h M L H 1$ or $h M S H 2$. This was first shown in colorectal cancers through the discovery that one of the commonest forms of clearly inherited susceptibility was due to germline mutations in one or other of these mismatch repair genes. Individuals carrying such a mutation in the heterozygote state do not show increases in mutation rates in lymphoblastoid cell lines derived from their normal lymphocytes compared with noncarriers of mismatch mutations. Their cancers, however, do show an increased mutation rate, as expected from the loss of mismatch repair function in their tumors, resulting from loss of the wild-type version of their mutated mismatch repair gene. Approximately $10 \%$ to $20 \%$ of sporadic colorectal cancers are also mismatch repair deficient, often due to epigenetic silencing of the $h M L H 1$ gene, sometimes in association with mutations in this gene. If this change were selected for because of its effect on the mutation rate, then one would expect that it occurred early because that is the time when the mutation rate is necessarily limiting, namely when the population size of the tumor is smallest. However, it has been shown that mismatch repair mutations do not occur before APC mutations in sporadic colorectal cancers (8). Tumors relatively rapidly reach sizes of $\sim 1 \mathrm{~cm}^{3}$, which corresponds to $\sim 10^{9}$ cells, within which there will be mutations in almost every gene, given reasonable assumptions about the normal mutation rate.

\section{Only a limited range of repair mutations is found in tumors}

Excision repair mutations, which are well-known to give rise to recessively inherited cancer susceptibility, rarely, if ever, occur in sporadic cancers. Missense variants in the base excision repair gene $M Y H$ have been shown to be associated with a form of familial polyposis that is recessive (or due to noncomplementing heterozygotes; ref. 9). However, $M Y H$ mutations are not found as somatic mutations in sporadic cancers. These observations strongly imply that there is no simple association between increased mutation rates and tumorigenesis.

If the mutations were there purely because of their effect on mutation rate, then one would expect to find a much wider range of types of such mutations in cancers. DNA repair mutations are, moreover, nearly always recessive. That means that two mutational events are required before there is any increase in mutation rate. Only then can any advantage of this increase be expressed through a higher rate of production of genetic mutations that are directly selected for. Under the basic assumption that it is only the increased instability that is selected for and not any direct selective effect of the mutated repair gene, these have to accumulate in the incipient tumor by chance, whereas any mutation with a direct selective effect, for example, on growth rate or independence, will immediately increase because of that selective effect. It can be shown theoretically, under very reasonable assumptions, that in that case, selectively advantageous mutations will spread through the tumor before there has been much of a chance for two repair mutations that lead to an increased mutation rate to occur (10).

In considering the effects of increased mutation rates on cancers, it is very important to distinguish somatic from germline mutations, such as occur in the DNA repair deficiency syndromes. The latter are almost always recessive, are found necessarily in every cell in the body, and lead to a systemic increase in the mutation rate, namely an increase in every cell in the body. Clearly, an overall increased mutation rate, or one that is influenced by a wide 
spread environmental mutagen such as sunlight, is expected to lead to an increased cancer incidence because it will enormously increase the risk of initiating mutations for a cancer.

\section{Tumor suppressor mutations are not really recessive in tumors}

Knudson's famous hypothesis was that there should be a relationship between germline and somatic cancer-related mutations (11). He proposed that a germline mutation in one copy of a gene that acted recessively in a tumor could give rise to an inherited susceptibility to cancer. This is because only one further event, namely another mutation in the same gene, would be needed somatically to create the recessive condition in the tumor cells that gives them their advantage. Subsequently, it has been shown that most, probably all, so-called tumor suppressors are not truly recessive in their action on a cancer. The first event already has some advantage, even if it is just a reduction of the gene dosage, and so gene activity, by half, namely "haploinsufficiency." Occasional tumors, for example, are found with a single change in a MMR gene, usually due to "hemimethylation," and so without MMR deficiency, suggesting that just reducing the level of hMLH1 product by a factor of two is enough to give the tumor some selective advantage (12).

Evidence for an advantage for the first $A P C$ mutation comes from the observation of a specific relation between the first and second APC mutations (13). About $60 \%$ of the somatic mutations cluster near the middle of the $A P C$ gene at a critical point for the function of the APC protein, and it seems that mutations in that region confer a greater selective advantage for the outgrowth of a tumor than mutations elsewhere, but this advantage is only fully expressed when the remaining wild-type allele is mutated or lost (see 14).

The carboxy terminus of APC contains a binding site for the end-binding protein EB1 that is closely associated with the centromere and the mitotic spindle. There is, thus, some evidence to suggest that $A P C$ mutations may contribute to chromosomal instability. However, MMRdefective colorectal cancers, which are mostly near diploid, do not become grossly chromosomally abnormal even if they are also mutated in the $A P C$ gene. In addition, mice with $A P C$ mutations that are defective in this $\mathrm{COOH}$-terminal function of the APC protein do not produce tumors in the gut (15). Thus, although this function of the $A P C$ gene may certainly be of biological importance, it does not seem to contribute to the early selection for $A P C$ mutations in colorectal cancers.

There is considerable evidence for some dominant effect of the first TP53 mutation. The high proportion of protein-producing mutations suggests a dominant (negative) effect (16).

Thus, at least in the case of $A P C, T P 53$, and probably $h M L H 1$, there is clear evidence for a selective advantage of the first mutation, although selection nearly always leads subsequently to complete loss of the normal function of the gene.

\section{Not all cancers are chromosomally unstable}

Chromosomal instability does not just refer to the occurrence of one or two abnormal chromosomes, nor to loss of heterozygosity. It means a grossly abnormal, and usually quite variable, karyotype, whose genetic basis is mostly not yet understood. By this definition, by no means are all cancers chromosomally unstable. For example, many leukemias and lymphomas are chromosomally stable and have only a limited number, often only one, abnormal chromosome(s), as in the classic case of chronic myelogenous leukemia. Although most carcinomas eventually have clear chromosomal instability, not all do. Notably, MMR deficient colorectal tumors are mostly stable and near diploid (17), and occasional other tumors are also. 


\section{Chromosomal instability is not found in adenomas}

Chromosomal instability is mostly not found in early precancerous growths, such as in colorectal adenomas, which are the precursors of nearly all colorectal cancers $(6,18)$. If instability were critical for the development of a tumor, it would be expected to occur early.

\section{Epigenetic Changes}

It was suggested many years ago that, in addition to mutation, relatively stable epigenetic changes could also be selected for in tumor evolution (see 19). The one known and much studied epigenetic mechanism is methylation (20). Depending on the mechanism, methylation may occur independently on each strand of the DNA or coordinately on both strands at more or less the same time. In the first case, it is quite analogous to mutation but probably occurs at a somewhat higher rate. Coordinate methylation changes could be influenced by various stress factors, including inflammatory responses (see 21). Methylation will only be a selective mechanism if either reducing the amount of gene product or eliminating it is the basis for the selection.

Although it has been suggested that there may be cancer "methylator" phenotypes, perhaps analogous to mutator phenotypes, our analysis of the methylation of several genes in colorectal cancer-derived cell lines does not support this idea. ${ }^{1}$ The methylation patterns, which do show correlations between the methylation changes in different genes, seem to be just as might be expected for an evolutionary process in which methylation is an alternative to mutation (see 3).

\section{Environmental Effects on Cancer}

There are, as might be expected, well-known environmental factors influencing cancer incidence that clearly involve major mutagenic effects, notably smoking, ionizing radiation, sunlight, and possibly viruses. However, it seems likely that other known environmental effects, especially diet, hormonal factors, and probably Helicobacter, are most probably not due to mutagens. The most striking evidence for this in the case of colorectal cancer is the lack of the sort of signature in the mutational spectra for APC and TP53 mutations that would be expected from obvious environmental mutagens $(22,23)$. The contrast with lung cancer in the incidence of $\mathrm{G}$ to $\mathrm{A}$ transversions is very clear, and it was the unusual incidence of such transversions in APC mutations in cancers from unexplained polyposis families, which led to the discovery of MYH variants as a basis for recessive polyposis (9).

\section{Conclusions}

Why are somatic mutations found in sporadic tumors in repair and related genes, such as TP53, and why is there so often chromosomal instability in sporadic cancers if the instability itself is not the primary driving force?

The answer almost certainly lies in the relation between repair or chromosomal instability, and apoptosis. Selection in tumors against apoptosis is as important, if not more so, than selection for increased growth rate (see 24). Each repair pathway seems to have a default line to apoptosis, in case the magnitude of DNA damage is excessive. This is also true for the control of chromosomal integrity during cell division. For TP53 mutations, it has become clear that a major selective effect is against apoptosis, presumably by some sort of dominant interference with the pathway from p53 to apoptosis. The same is likely to be the

\footnotetext{
${ }^{1}$ O. Dikomitou, J. Wilding, Y. Liu, W. Bodmer, unpublished observations.
} 
case for $h M L H 1$ and $h M S H 2$ mutations, and can be presumed to be likely for aspects of the control of chromosomal integrity, other than by p53.

Selection against apoptosis could account for the reason why only a small subset of genes involved in repair is found to be mutated somatically in tumors. Only that subset may give rise to interference with the apoptotic pathways and so to the selective advantage, initially in the heterozygote, which accounts for their presence in many cancers.

It has been claimed that the overall number of mutations seen in cancers, whether functionally relevant or not, cannot be accounted for without an increase in the mutation rate. However, based on quite reasonable assumptions about the normal mutation rate and the cell biology of cancer development, it can be shown that this is not the case (25).

The key issue is that mutations that provide a selective advantage are essential for tumorigenesis. The direct selective effects of such mutations override the secondary effects of mutations that increase genomic instability and only indirectly contribute to the somatic evolution of cancers. Genomic instability may, however, often be a byproduct of direct selective effects. It will then, in at least some cases, have important consequences for the subsequent evolutionary behavior of a cancer.

Most sporadic tumors start to grow with a normal mutation rate as they accumulate their successive advantageous mutations leading to clonal expansion. Although some tumors will acquire a mutator phenotype before they present clinically, it is not necessary to invoke an increased mutation rate to explain the development of cancers.

\section{References}

1. Fearon ER, Vogelstein B. A genetic model for colorectal tumorigenesis. Cell. 1990; 61:759-67. [PubMed: 2188735]

2. Bodmer WF. The somatic evolution of cancer. The Harveian Oration of 1996. J R Coll Physicians Lond. 31:82-9. [PubMed: 9044206]

3. Bodmer WF. Cancer genetics: colorectal cancer as a model. J Hum Genet. 2006; 51:391-6. [PubMed: 16596323]

4. Loeb LA. Mutator phenotype may be required for multistage carcinogenesis. Cancer Res. 1991; 51:3075-9. [PubMed: 2039987]

5. Lengauer C, Kinzler KW, Vogelstein B. Genetic instabilities in human cancers. Nature. 1998; 396:643-9. [PubMed: 9872311]

6. Sieber OM, Heinimann K, Tomlinson IPM. Genomic instability - the engine of tumorigenesis? Nat Rev Cancer. 2003; 3:701-8. [PubMed: 12951589]

7. Fisher, RA. The Genetical Theory of Natural selection. 2nd ed.. New York: Dover (1st Ed. Oxford Univ Press); 1930.

8. Homfray TF, Cottrell TFSE, Ilyas M, et al. Defects in mismatch repair occur after APC mutations in the pathogenesis of sporadic colorectal tumours. Hum Mutat. 1998; 11:114-20. [PubMed: 9482574]

9. Al-Tassan N, Chmiel NH, Maynard J, et al. Inherited variants of MYH associated with somatic G:C3T:A mutations in colorectal tumors. Nat Genet. 2002; 30:227-32. [PubMed: 11818965]

10. Tomlinson IP, Novelli MR, Bodmer WF. The mutation rate and cancer. Proc Natl Acad Sci U S A. 1996; 93:14800-3. [PubMed: 8962135]

11. Knudson AG. Mutation and cancer statistical study of retinoblastoma. Proc Natl Acad Sci U S A. 1971; 68:820-3. [PubMed: 5279523]

12. Wheeler JM, Loukola A, Aaltonen LA, Mortensen NJ, Bodmer WF. The role of hypermethylation of the $h M L H 1$ promoter region in HNPCC versus MSI+ sporadic colorectal cancers. J Med Genet. 2000; 37:588-92. [PubMed: 10922385] 
13. Rowan AJH, Lamlum M, Ilyas J, et al. $A P C$ mutations in sporadic colorectal tumors: a mutational 'hotspot' and interdependence of the 'two hits'. Proc Natl Acad Sci U S A. 2000; 97:3352-7. [PubMed: 10737795]

14. Fearnhead NS, Britton MP, Bodmer WF. The ABC of APC. Hum Mol Genet. 2001; 10:721-33. [PubMed: 11257105]

15. Nathke I. Cytoskeleton out of the cupboard: colon cancer and cytoskeletal changes induced by loss of APC. Nat Rev Cancer. 2006; 6:967-74. [PubMed: 17093505]

16. Liu Y, Bodmer WF. Analysis of p53 mutations and their expression in 56 colorectal cancer cell lines. Proc Natl Acad Sci U S A. 2006; 103:976-81. [PubMed: 16418264]

17. Muleris M, Dutrillaux AM, Olschwang S, Salmon RJ, Dutrillaux B. Predominance of normal karyotype in colorectal tumors from hereditary non-polyposis colorectal cancer patients. Genes Chromosomes Cancer. 1995; 14:223-6. [PubMed: 8589041]

18. Sieber OMK, Heinimann P, Gorman H, et al. Analysis of chromosomal instability in human colorectal adenomas with two mutational hits at $A P C$. Proc Natl Acad Sci U S A. 2002; 99:169105. [PubMed: 12486240]

19. Bodmer W. Somatic cell genetics and cancer. Cancer Surv. 1988; 7:239-50. [PubMed: 3066473]

20. Jones PA, Baylin SB. The fundamental role of epigenetic events in cancer. Nat Rev Genet. 2002; 3:415-28. [PubMed: 12042769]

21. Wong NACS, Wilding JL, Bartllett S, et al. CDX1 is an important molecular mediator of Barrett's metaplasia. Proc Natl Acad Sci U S A. 2005; 102(21):7565-70. Epub 2005 May 13. [PubMed: 15894614]

22. Harris RA. p53 - at the crossroads of molecular carcinogenesis and risk assessment. Ann N Y Acad Sci. 1993; 262:1980-1.

23. Bodmer W. Familial adenomatous polyposis (FAP) and its gene, APC. Cytogenet Cell Genet. 1999; 86:99-104. [PubMed: 10545698]

24. Johnston MD, Edwards CM, Bodmer WF, Maini PK, Chapman SJ. Examples of mathematical modeling: tales from the crypt. Cell Cycle. 2007; 6:216-12. Epub 2007 Jun 27. [PubMed: 17873520]

25. Tomlinson I, Sasieni P, Bodmer W. How many mutations in a cancer? Am J Pathol. 2002; 160:755-8. [PubMed: 11891172] 